\title{
Espacio-tiempo Anti-de Sitter (AdS)
}

\author{
Fulgencio Villegas ${ }^{* 1}$ \\ ${ }^{1}$ Universidad Nacional Mayor de San Marcos, Facultad de Ciencias Físicas, Lima, Perú
}

Recibido 30 noviembre 2019 - Aceptado 27 diciembre 2019

\begin{abstract}
Resumen
En este trabajo se hace una introducción básica acerca de los espacios- tiempo de Sitter y Anti-de Sitter. Para el espacio de Sitter se calcula dos métricas equivalentes una usando coordenadas estáticas y la otra usando coordenadas de Friedmann-Lemaître-Robertson-Walker (FLRW). En el caso del espacio Anti-de Sitter se determina la condición que debe cumplir la constante cosmológica para ser una solución de las ecuaciones de Einstein y finalmente, haciendo uso de coordenadas globales se determina su métrica.

Palabras clave: Relatividad general, gravitación, cosmología, Anti-de Sitter.
\end{abstract}

\section{Anti-de Sitter (AdS) spacetime}

\begin{abstract}
In this work a basic introduction is made about the space-time of Sitter and Anti-Sitter. For the Sitter space, two equivalent metrics are calculated, one using static coordinates and the other using FriedmannLemaitre-Robertson-Walker coordinates (FLRW). In the case of the Anti-Sitter space, the condition that the cosmological constant must meet is determined to be a solution of Einstein's equations and finally, using global coordinates, its metric is determined.
\end{abstract}

Keywords: General Relativity, gravitation, cosmology, Anti-de Sitter..

\section{Introducción}

El espacio-tiempo de Sitter (dS) y Anti de Sitter (AdS) son soluciones de las ecuaciones de campo de Einstein en ausencia de materia. El espacio de Sitter considera una constante cosmológica $(\Lambda)$ positiva, por lo tanto incluye una fuerza repulsiva. El espacio de Sitter cumple un papel fundamental en la cosmología moderna; por un lado, esta geometría se aproxima al fenómeno de expansión exponencial del universo primordial en la época de inflación [San19].

Por otro lado, la geometría de Sitter resulta ser la referencia ante el fenómeno de expansión acelerada del universo [Bah19]. Tambien es importante analizar un modelo donde la cosntante cosmológica sea negativa; pues el espacio-tiempo AdS resulta de considerar una cosntante cosmológica negativa. El espacio-tiempo AdS ha sido un argumento enigmático e interesante de investigación, en sus inicios como un modelo cosmológico y posteriormente como una herramienta importante por el papel que juega en la correspondencia AdS/CFT y en el principio holográfico [Sor19].

*fvillegass@unmsm.edu.pe

\section{Espacios máximamente simétricos}

Un espacio es máximamente simétrico si es que presenta el máximo numero de isometrías; es decir, si tiene el mayor número de vectores de Killing.

Para entender mejor acerca de los espacios máximante simétricos veamos algunas definiciones.

Definición 1. Dada una variedad $(M, g)$, se dice que es homogénea si para cada par de puntos $p, q \in M$ existe una isometría global $A: M \longrightarrow M$ tal que $A(p)=q$.

Esto significa que, en una variedad homogénea, todos los puntos de la variedad son indistinguibles por ser equivalentes; es decir la métrica presenta tanta simetría que permite relacionar dos puntos cualesquiera a través de una relación de simetría. por tanto, la homogeneidad es una propiedad global de una variedad.

Definición 2. Dada una variedad $(M, g)$, se dice que es isótropa en un punto $p \in M$ si dados $v, w \in T_{p} M$ existe una isometría $A: T_{p} M \longrightarrow T_{p} M$ tal que $A(v)=w$.

Esto significa que, en un espacio isotrópico, no existe ninguna dirección privilegiada; por tanto, las superficies 
isótropas tienen la misma forma en todas direcciones. Esto se puede interpretar como que la métrica es esféricamente simétrica. Por tanto, la isotropía no es un concepto global.

Definición 3. Una variedad $(M, g)$ que es homogénea e isótropa se dice que es máximamente simétrica, es decir, tiene el máximo número de simetrías.

En general, podemos concluir que las variedades máximamente simétricas son espacios con curvatura constante. Existen algunas relaciones importantes respecto a la curvatura para espacios máximamente simétricos [Bla19].

1. El escalar de curvatura $R$ es constante

2. El tensor de Ricci es proporcional a la métrica, $R_{\mu \nu}=\frac{1}{n} R g_{\mu \nu}$

3. El tensor de Riemann está dado por $R_{\mu \nu \rho \sigma}=$ $\frac{R}{n(n-1)}\left(g_{\mu \rho} g_{\nu \sigma}-g_{\nu \rho} g_{\mu \sigma}\right)$

Estas relaciones fueron estudiadas por primera vez por Élie Cartan [Bes07.

\section{Universo de Sitter (dS)}

Wilhem de Sitter en 1917 [Sit16] planteó una solución a las ecuaciones de Einstein considerando una constante cosmológica y en un universo sin masa. Entonces, las ecuaciones de campo gravitatorio de Einstein toman la forma:

$$
R_{\mu \nu}-\frac{1}{2} g_{\mu \nu} R+\Lambda g_{\mu \nu}=0,
$$

donde $R_{\mu \nu}$ es el tensor de Ricci y $\Lambda$ es la constante cosmológica. En la ecuación (1) aplicando la traza, obtenemos

$$
R=4 \Lambda
$$

Sustituimos el valor del escalar de curvatura de Ricci en las ecuaciones de campo de Einstein se obtiene [Lor74]

$$
R_{\mu \nu}=\Lambda g_{\mu \nu}
$$

Definición 4. Una variedad de Einstein es una variedad riemanniana o pseudo-riemanniana si cumple con la ecuación (3).

La métrica más sencilla del universo de dS se puede expresar en coordenadas estáticas. Es decir, que los coeficientes de esta métrica no dependen del tiempo y sean esféricamente simétricos.

Planteamos un modelo (ansatz) de la forma:

$$
d s^{2}=-e^{2 A(r)} d t^{2}+e^{2 B(r)} d r^{2}+r^{2} d \theta^{2}+r^{2} \operatorname{sen}^{2} \theta d \phi^{2}
$$

Haciendo uso de la ecuación (3) obtenemos la siguiente métrica:

$$
d s^{2}=-\left(1-\frac{\Lambda}{3} r^{2}-\frac{2 M}{r}\right) d t^{2}+\left(1-\frac{\Lambda}{3} r^{2}-\frac{2 M}{r}\right)^{-1} d r^{2}+r^{2} d \Omega^{2} .
$$

Esta métrica fue obtenida inicialmente por de Sitter y describe el agujero negro de Schwarzschild cuando $\Lambda \longrightarrow 0$. Haciendo $M=0$ se obtiene la métrica de Sitter en coordenadas estáticas; por tanto, a esta métrica se la conoce como la métrica de Schwarzschild-de Sitter.

Haciendo $M=0$ en la métrica dada por la ecuación (5), el tensor de Riemann y el tensor de Ricci toman las formas

$$
\begin{gathered}
R_{\mu \nu \rho \lambda}=-R_{0}^{-2}\left(g_{\mu \lambda} g_{\nu \rho}-g_{\mu \rho} g_{\nu} \lambda\right) \\
R_{\rho \lambda}=\frac{3}{R_{0}^{2}} g_{\rho \lambda}
\end{gathered}
$$

donde $R_{0}$ es el radio de de Sitter. Haciendo uso de la ecuación (3) determinamos el radio de de Sitter

$$
R_{0}=\sqrt{\frac{3}{\Lambda}}
$$

Reemplazando en la ecuación (5) para el caso $M=0$ obtenemos Kim03.

$$
d s^{2}=-\left(1-\frac{r^{2}}{R_{0}^{2}}\right) d t^{2}+\left(1-\frac{r^{2}}{R_{0}^{2}}\right)^{-1} d r^{2}+r^{2} d \Omega^{2}
$$

La expresión anterior es la métrica que describe el espaciotiempo de de Sitter en coordenadas estáticas.

Existe otra forma de calcular la métrica del universo de de Sitter, consiste en usar las coordenadas de FriedmannLemaître-Robertson-Walker (FLRW); esto es, se propone un anzat con la forma de la métrica de FLRW

$$
d s^{2}=-d t^{2}+e^{2 A(t)} \delta_{i j} d x^{i} d x^{j}
$$

donde $e^{2 A(t)}=a(t)$ es el factor de escala.

Calculando el tensor de Ricci y haciendo uso de la ecuación (3) se determina el valor de $A(t)$ dado por

$$
A(t)=\sqrt{\frac{3}{\Lambda}} t=R_{0}^{-1} t
$$

Finalmente obtenemos la métrica de de Sitter en coordenadas FLRW, la que presenta la forma siguiente

$$
d s^{2}=-d t^{2}+e^{2 t / R_{0}} \delta_{i j} d x^{i} d x^{j}
$$

Cabe indicar que las métricas dadas por las ecuaciones (9) y (12) son equivalentes.

Un espacio-tiempo de de Sitter de 4-dimensiones puede ser definido por aplicación en un espacio plano de Minkowski 
de (4+1)-dimensiones que corresponde a un hiperboloide (Figura 1) dado por:

$$
\sum_{i=1}^{4}\left(x^{i}\right)^{2}-\left(x^{0}\right)^{2}=R^{2}
$$

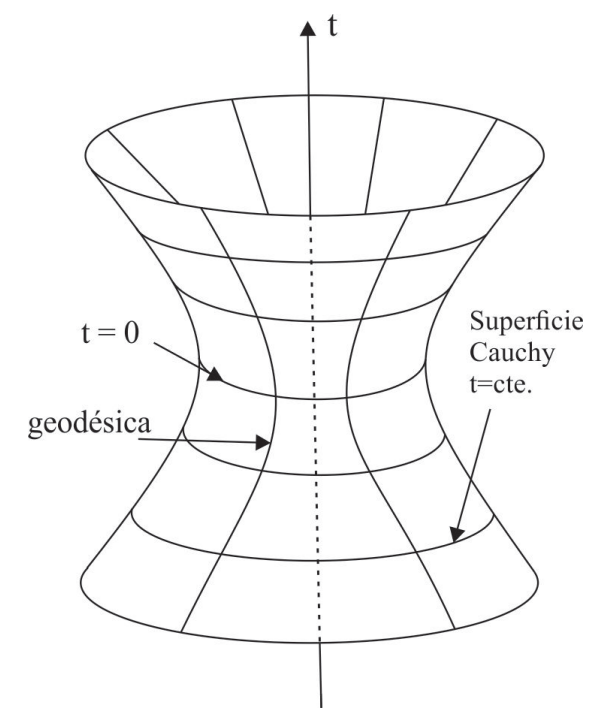

Figura 1: Visualización de dS como un hiperboloide.

El espacio-tiempo de de Sitter puede ser visualizado como una hipersuperficie tetradimensional inmerso en un espacio pseudo-euclidiano lorenziano de cinco dimensiones.

\section{El espacio de Anti- de Sitter (AdS)}

El espacio-tiempo Anti- de sitter (AdS) es una solución máximamente simétrica de las ecuaciones de campo de Einstein sin materia pero con constante cosmológica negativa.

El espacio-tiempo AdS se puede definir como una superficie cuadrática en un espacio-tiempo plano de $d+1$ dimensiones:

$$
\left(\chi^{1}\right)^{2}+\left(\chi^{2}\right)^{2}+\ldots+\left(\chi^{d+1}\right)^{2}=L^{2} .
$$

El espacio-tiempo Ads es una solución interesante de la materia libre a las ecuaciones de campo de Einstein debido a que posee la máxima cantidad de simetrías, para el caso de $\Lambda=0$ obtenemos el espacio de Minkowski.

El espacio-tiempo AdS no colapsa y es un espacio de curvatura constante negativa. El modelo AdS no se toma en serio como un modelo cosmológico, pero se usa como herramienta matemática en física teórica y cumple un papel fundamental en la teoría de holografía y en la conjetura de Maldacena; también es importante por que permite entender mejor la naturaleza de la constante cosmológica; pues el espacio-tiempo AdS posee una rica estructura debido a la presencia de una simetría de aproximación asintótica, la cual debe cumplir con las siguientes condiciones:

1. La condición asintotica debe ser invariante bajo la acción del grupo AdS como de otra transformación asintótica.

2. Las transformaciones simétricas deben tener bien definidos los generadores canónicos.

3. Las condiciones límite deben incluir las soluciones asintóticas AdS como la métrica de Kerr Ads.

4. Las condiciones límite deben ser expresadas en términos de las componentes de la métrica.

Haciendo uso de la ecuación de Einstein para la Relatividad General con $D=d+1$ dimensiones calcularemos la condición que debe cumplir $\Lambda$ para que AdS sea una solución de la ecuación de Einstein. Para los espacios máximamente simétricos el tensor de Riemann podemos escribirlo como:

$$
R_{a b c d}=k\left(g_{a c} g_{b d}-g_{a d} g_{b c}\right)
$$

donde $k$ es una constante que es función del escalar de curvatura y para el espacio AdS consideramos $k=-1 / L^{2}$, siendo $L$ el radio del espacio AdS.

El tensor de Ricci viene dado por $R_{a c}=g^{b d} R_{a b c d}$, de donde obtenemos que:

$$
R_{a c}=-\frac{1}{L^{2}}(D-1) g_{a c}
$$

siendo $D$ el número de dimensiones del espacio AdS. El escalar de Ricci o escalar de curvatura viene dado por:

$$
R=g^{a c} R_{a c}=-\frac{D(D-1)}{L^{2}} .
$$

Reemplazando las ecuaciones (16) y (17) en la ecuación (1), se tiene:

$$
\begin{aligned}
-\frac{1}{L^{2}}(D-1) g_{a c}+\frac{1}{2} g_{a c} \frac{D(D-1)}{L^{2}}+\Lambda g_{a c} & =0 \\
\frac{(D-1)}{L^{2}} g_{a c}\left(-1+\frac{D}{2}\right) & =-\Lambda g_{a c},
\end{aligned}
$$

de donde obtenemos que:

$$
\Lambda=-\frac{(D-1)(D-2)}{2 L^{2}}
$$

Esta relación es la concidión que debe satisfacer $\Lambda$ para que AdS sea una solución de la ecuación de Einstein. Ahora introduciremos $A d S_{d+1}$ como una superficie inmersa o embebida en $\mathbb{R}_{2 d}$. Por tanto $A d S_{d+1}$ estará definido por el hiperboloide:

$$
-X_{-1}^{2}-X_{0}^{2}+X_{1}^{2}+\ldots+X_{d}^{2}=-L^{2},
$$

en un espacio de $(d+2)$ dimensiones cuya métrica plana es

$$
d s^{2}=-d X_{-1}^{2}-d X_{0}^{2}+d X_{1}^{2}+\ldots+d X_{d}^{2} .
$$


Haciendo el siguiente cambio de coordenadas llamado parche de Poincare:

$X_{-1}=\frac{Z}{1}\left(1+\frac{1}{Z^{2}}\left(1+x^{i} x^{i}-t^{2}\right)\right), X_{0}=\frac{L}{z} t, X^{i}=$ $\frac{L}{z} x^{i}, X_{d}=\frac{Z}{1}\left(1-\frac{1}{Z^{2}}\left(1+x^{i} x^{i}-t^{2}\right)\right)$ con $i=1, \ldots, d$, entonces el elemento de línea de $A d S_{d+1}$ lo podemos escribir del siguiente modo:

$$
d s^{2}=\frac{L^{2}}{z^{2}}\left(\eta_{\mu \nu} d x^{\mu} d x^{\nu}+d z^{2}\right)
$$

Por otro lado, también podemos hacer uso de las coordenadas globales dadas por: $X_{-1}=L \cosh \rho \cos \tau, X_{0}=$ $L \cosh \rho \operatorname{sen} \tau, X^{i}=L \operatorname{senh} \rho \hat{a}^{i}$, obtenmos:

$$
d s^{2}=L^{2}\left(-\cosh ^{2} \rho d \tau^{2}+d \rho^{2}+\operatorname{senh}^{2} \rho d \Omega_{d-1}^{2}\right)
$$

Haciendo la transformación de coordenadas: $r=$ $L \operatorname{senh} \rho$ y $t=L \tau$, finalmente obtenemos una métrica, en coordenadas globales, para $A d S_{d+1}$ de la forma Nat15:

$$
d s^{2}=-\left(1+\frac{r^{2}}{L^{2}}\right) d t^{2}+\left(1+\frac{r^{2}}{L^{2}}\right)^{-1} d r^{2}+r^{2} d \Omega_{d-1}^{2}
$$

Notamos que $A d S_{d+1}$ en el parche de Poincaré presenta una divergencia para $z=0$ llamado "borde". Considerando un valor pequeño para $z \longrightarrow \varepsilon$ con $\varepsilon \ll 1$ la métrica toma la forma

$$
d s^{2}=\frac{L^{2}}{\varepsilon^{2}}\left(\eta_{\mu \nu} d x^{\mu} d x^{\nu}\right)
$$

Ahora analizamos la siguiente transformación conforme

$$
d s^{2}=\Omega(x) d s^{2}
$$

de tal manera que $d s^{2}$ no presente singularidad cuando $\varepsilon \longrightarrow 0$, entonces el elmento de línea $d s^{, 2}$ es el espacioo de Minkowski $M_{d}$. Entonces el borde de $A d S_{d+1}$ se convierte en un borde conforme; a la teoría de campos en el borde se le conoce como la teoría del borde y a la teoría de la gravedad como la teoría del bulk.

Otra manera de analizar de que $A d S_{d+1}$ presenta un borde es recurriendo a su diagrama de Penrose en coordenadas globales. Si factorizamos el término $\left(1+r^{2} / L^{2}\right)$ en la métrica (23) y efectuamos el cambio de variable $d \rho=d r /\left(1+r^{2} / L^{2}\right)$ considerando $L=1$, la métrica de $A d S_{d+1}$ toma la forma:

$$
d s^{2}=\left(1+r^{2}(\rho)\right)\left(-d t^{2}+R^{2}(\rho) d \Omega_{d-1}^{2}+d \rho^{2}\right)
$$

donde $R^{2}(\rho)$ es una función positiva que determina el radio de la esfera $S^{d+1}$. Entonces el diagrama de Penrose de $A d S_{d+1}$ es un cilindro sólido de altura infinita Gri12. como se muestra en la Figura 2

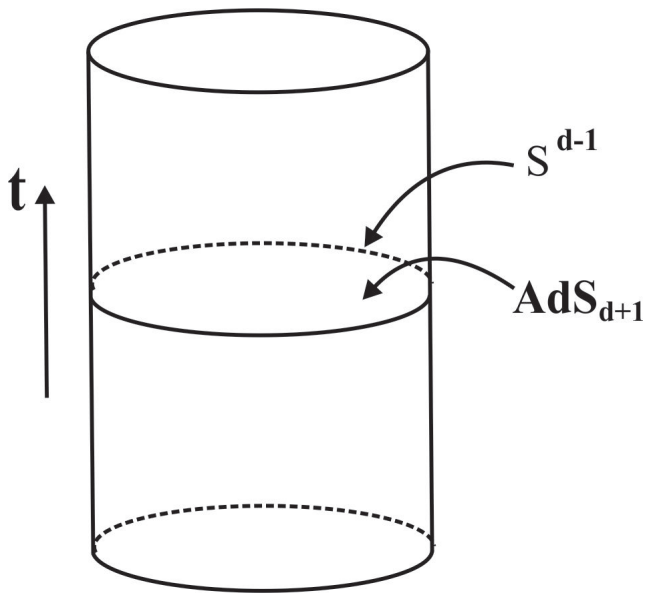

Figura 2: Diagrama para el espacio $A d S_{d+1}$.

Por lo tanto, en el espacio-tiempo AdS la constante cosmológica se comporta como un potencial atractivo. Es decir, la constante cosmológica negativa nos reproduce un diagrama conforme que en el formalismo de Penrose resulta ser un cilindro sólido.

\section{Conclusiones}

El espacio-tiempo de Sitter resulta de la solución de las ecuaciones de campo de Einstein sin fuentes y en presencia de una cosntante cosmológica. Para el espacio de Sitter se han determinado dos métricas, una en coordenadas estáticas y la otra en coordenadas tipo FLRW siendo ambas equivalentes. Uno de los puntos fundamentales para estudiar la métrica de Sitter es la evidencia cosmológica en favor de $\Lambda>0$. Llama la atención este espacio que a pesar que representa una solución del vacío es dinámico; por tanto, es importante el estudio del espacio-tiempo de Sitter ya que nos permite analizar el proceso de expansión exponencial del universo en su etapa primordial.

El espacio-tiempo anti-de Sitter es máximamente simétrico; es decir, es la solución de vacío conformalmente plana con una constante cosmológica negativa. Por lo tanto, tiene una curvatura escalar constante y es el complemento del espacio plano de Minkowski y del espaciotiempo de Sitter. Se ha determinado la condición que debe cumplir la constante cosmológica para ser una solución de las ecuaciones de campo de Einstein, la cual se ha demostrado es inversamente proporcional al cuadrado del radio del espacio AdS.

Realizando un embebimiento de $A d S_{d+1}$ en un espacio $\mathbb{R}_{2 d}$ se ha conseguido calcular la métrica $A d S$ y mediante un cambio de coordenadas adecuadas se ha logrado remover la singularidad en el borde. Cabe indicar que el estudio del espacio-tiempo $A d S$ ha cobrado importancia recientemente en el contexto de las teorías de dimensiones superiores, en particular en el estudio de la dualidad AdS/CFT y en la teoría de cuerdas. 


\section{Referencias}

[Bah19] Bahamonde, S., Marciu, M., Rudra, P. (2019). Dynamical system analysis of generalized energymomentum-squared gravity. Phys. Rev. D 100, 083511.

[Bes07] Besse, A. (2007). Einstein manifolds, SpringerVerlag, Classics in Mathematics Series.

[Bla19] Blau, M. (2019). Lecture Notes on General Relativity, Institut für Theoretische Physik, Universität Bern, Switzerland.

[Gri12] Griffiths, J., Podolský, J. (2012). Exact spacetimes in Einstein's general relativity, Cambridge Monographs on Mathematical Physics (1st edn.), Cambridge University Press.

[Kim03] Kim, Y., Young, C. and Park, N. (2003). Classical Geometry of De Sitter Spacetime: An Introductory Review. Journal of the Korean Physical Society, vol 42, No. 5.
[Lor74] Lord, E. (1974). Redshift Formulae for De-Sitter Type Metrics. International Journal of Theoretical Physics, vol 9, No. 2.

[Nat15] Makoto Natsuume (2015). AdS/CFT Duality User Guide, Springer-Verlag, Lecture Notes in Physics 903.

[San19] Sanchez, N. (2019). New quantum phase of the Universe before inflation and its cosmological and dark energy implications. Int. J. Mod. Phys. A 34 1950155 .

[Sit16] De Sitter, W. (1916). On Einstein's theory of gravitation and its astronomical consequences, Second Paper, §30, Mon. Not. R. Astron. Soc. 77, 181-184 (disponible en: http://articles .adsabs . harvard.edu).

[Sor19] Sorce, J. (2019). Holographic entanglement entropy is cutoff-covariant. Journal of High Energy Physics, 10. 
